\title{
General Chemistry
}

Donald A. McQuarrie and Peter A. Rock

Donald A. McQuarrie and Peter A. Rock

Thoroughly revised and updated, this major

textbook for mainstream chemistry courses,

focuses on principles while interweaving the most

important facets of descriptive chemistry. It is

now avallable, for the first time, as an

international student Edition.

f42.95: ISE, f19.95

\section{Quantitative Chemical Analysis}

Daniel C. Harris

Written in a clear, informal style that will appea

to students and instructors alike, this text is

designed for students with a background in

general chemistry who are preparing for work in

general chemistry who are preparing for work

advanced studies in chemistry.

advanced studies in chemistry.

E21.95

\section{Universe}

William J. Kaufmann III

Thoroughly updated, the Third Edition of this text still offers an outstanding full-colour illustration programme, and has retained its highly acciaimed exposition, noted for its clarity and a sense of excitement.

January 1991, 656 pages, 710 illus, board, f27.95

One-dimensional and Two-

dimensional NMR Spectra by

Modern Pulse Techniques

Edited by Kojl Nakanish

This unique collection of selected spectra is a

suitable guide for professional NMR

spectroscopists and students. Using a picture

book format and minimal theory, each example is

accompanied by brief comments

November 1990, 234 pages, paper, f29.95

The Hour of Our Delight

Cosmic Evolution, Order, and Complexity

Hubert Reeves

Hubert Reeves, one of France's most

distinguished researchers and well-know

popularizer of science, examines the evolution of

our Universe, describing the increasingly

organized structure of matter and our own

species' climb up the ladder of complexity. The

Hour of Our Delight won the prestigious Prix

Blaise Pascal and is now published in English for

the first time

May 1991, 246 pages, 20 illus, board, $\mathbf{f} 14.95$

The Shadows of Creation

Dark Matlel and the Structure of the Universe

Michael Riordan and David N. Schramm

Foreword by Stephen Hawking

More than ninety percent of the Universe is

unseen, unknown, yet there is convincing

(hat it exists - as something called "dark

matter. Moin Michael Riordan and David

Schramm as they expiore this most remarkable

mystery of the decade, describing efforts

underway at observatories, laboratories, and

particle colliders to determine the nature of dark

matter

April 1991, 278 pages, 62 illus, board, $\mathbf{1} 15.95$

Fractals, Chaos, Power Laws

Manfred Schroeder

Fractals Chaos, Power Laws reveals the

fascinating consequences of new insights into the

realm of symmetry The reader will explore the

surprising presence of self-similar symmetries in

surprising presence of self-similar symmetries in

physics, chemistry, music, and the visual arts.

\section{Discovering Enzymes}

David Dressler and Huntington Potter

David Dressler and Huntington Potter convey the

excitement of scientific detective work as they

show how the attempt to understand alcoho is

fermentation led to the discovery of enzymes,

how the key to enzymatic function was

uncovered, and how enzymes are isolated and

studied at the molecular level.

studied at the molecular
Scientific American Library

January 1991, 263 pages, 150 illus, board, $£ 16.95$

The Science of Words

George A. Miller

The Science of Words offers both historical

perspective and future prospects for

psycholinguistics, while deepening our

understanding of the relations among words,

language, thought, and the human brain.

Scientific American Library

May 1991, 276 pages, 96 illus, board, $f 16.95$

For details about the latest keadings trom

Scientific American please call 0865.726975 or

write to the address below

DW H Freeman

\section{Nonlinear explanations}

Robert M. May

Fractals, Chaos, Power Laws: Minutes

from an Infinite Paradise. By Manfred Schroeder. Freeman: 1991. Pp.429. £24.95, \$32.95.

ONE of my ambitions is to write an essay on why some books about science - Hawking, Penrose, Gleick - succeed beyond all resonable expectation, whereas others languish. My problem is that, like the publishers themselves, I can explain these successes in retrospect better than in prospect. Easier to understand is the 'Jaws II' syndrome, whereby one book that has captured the popular imagination is followed by others that amplify the theme.

Which brings us to Fractals, Chaos, Power Laws: Minutes from an Infinite Paradise by Manfred Schroeder. The general level and style suggest that Schroeder is targeting an audience of mathematically literate scientists, aiming to go beyond James Gleick and others both in dealing with a wider range of topics and in treating them in greater depth. Although the title leads off with Fractals, Chaos, much of the emphasis is on scaling phenomena or Power Laws and on their pervasive occurrence in physics, chemistry, biology, music and elsewhere.

I found the book very engaging, but also very exasperating. Schroeder covers a vast amount of territory, much of it going beyond what previous books have made familiar. There are, for example, interesting discussions of scaling and symmetry in relation to "acousticians, alchemy, and concert halls", to Bach "composing on all scales", to trees, rivers, arteries, lungs, and stock markets. Old gambling paradoxes are discussed in relation to new themes. Some of the exposition is rather demanding, yet will reward those who persist: how to determine fractal dimensions from time series or fracture surfaces, or how to compute the dimension of a strange attractor.

The subtitle exemplifies the more exasperating aspects of the book. Minutes from an Infinite Paradise is a marvellous phrase, but I still have no clue how it relates to the book's contents. Far too many of the explanations are themselves nonlinear (you cannot understand any of it unless you understand all of it); some passages are excessively telegraphic, whereas others meander. For instance, there are 32 pages on the quadratic map, which go into some of the fascinating details of its cascades of period doubling and its ap-

- An Eye for Fractals: A Graphic and Photo graphic Essay by Michael McGuire has just been published by Addison-Wesley at $\$ 29.95$, £24.75. It contains 90 black and white photographs plus an annotated bibliography. parently chaotic behaviour. Despite the generous length, I found this discussion hard to follow, and the description of "chaos... interleaved with periodic windows" left me with the impression that the author does not really understand what is going on in this regime. Earlier popularizations of chaos have been criticized for giving insufficient credit to non-American workers. Schroeder discusses early Russian work, but does not mention the yet earlier work of the Finn Myrberg, nor of the French; given the abundance of references and other scholarly apparatus in this book, such omissions are less excusable than in earlier books.

At the telegraphic extreme is a chapter entitled 'Percolation: from Forest Fires to Epidemics', where the word epidemic is used in the third and the second to last paragraphs, but epidemics as such are never discussed. In the chapter on 'Noises: White, Pink, Brown, and Black', Schroeder refers to a recent paper by Redfearn and Pimm (see Nature 334, 613-615; 1988) as showing that fluctuations in terrestrial animal populations get larger as the observation interval lengthens. In fact, this paper has stimulated a lively correspondence, and its conclusions are by no means apodictic. Schroeder's point is, however, illustrated unambiguously by the pink noise spectrum characteristic of environmental fluctuations in the sea, which are not mentioned. These criticisms, and others that could be made, might look nitpicking, but - given the softness in the areas I know well - I am left wondering whether the book's admirably wide coverage has been achieved at the expense of consistent accuracy.

Schroeder was one of the first people to see the purely artistic possibilities inherent in computer graphics. Indeed, he won first prize at the International Computer Art Exhibition in 1969. The graphics in this book are thus excellent, and the inset of nine coloured figures would be stunning if earlier coffee-table chaos books and calenders had not already spoiled us. I especially like the illustration, and supporting analysis, of the fractal basins of attraction generated by Newton's iterative method applied to $z^{3}=1$. And those not already familiar with the Mandelbrot Set will enjoy the illustration of fat little Mandelbrots nested one within the other, an infinite regression that gives a kind of concreteness to pre-scientific visions of life not coded by DNA but rather unfolding from generation to generation through successive homunculi, nested like Russian dolls inside the egg.

More than other recent books in its general area, this book conveys a sense of the broad sweep of recent work on chaos, fractals and scaling laws. Read in this spirit, as an impressionistic guidebook and not as a map, it is fun.

Robert M. May is in the Department of Zoology, Oxford University, South Parks Road, Oxford, OX1 3PS, UK. 